\title{
Thyroid Involvement in Eosinophilic Granulomatosis with Polyangiitis
}

\author{
Salem Bouomrani ${ }^{1,2 *}$ \\ ${ }^{1}$ Department of Internal medicine, Military Hospital of Gabes, Tunisia \\ ${ }^{2}$ Sfax Faculty of Medicine, University of Sfax, Tunisia
}

Submission: June 24, 2020; Published: July 27, 2020

*Corresponding author: Salem Bouomrani, Department of Internal medicine, Military Hospital of Gabes, Gabes 6000, Tunisia.

\begin{abstract}
Thyroid disorders are among the most frequent endocrine pathologies with an overall prevalence estimated at $10 \%$. Autoimmunity plays a crucial role in most thyroid dysfunctions, which explains the frequent associations with other autoimmune diseases. Eosinophilic granulomatosis with polyangiitis (EGPA) (formerly Churg-Strauss syndrome) is a necrotizing systemic vasculitis of small-sized vessels specifically associated with antineutrophil cytoplasmic antibodies (ANCA). Thyroid involvement is exceptional and uncommon in association with systemic vasculitis. It has not been previously reported during EGPA. We discuss the possible theoretical mechanisms of thyroid dysfunction that can be seen during this systemic angiitis.
\end{abstract}

Keywords: Thyroid gland; Eosinophilic granulomatosis with polyangiitis; EGPA; Thyroiditis; ANCA; Vasculitis

\section{Introduction}

Eosinophilic granulomatosis with plyangitis (EGPA), formerly known as Allergic Granulomatous Angiitis or Churg-Straus Syndrome is a very rare necrotizing vasculitis of small and medium-sized systemic blood vessels [1,2]. It was individualized from periarteritis nodosa (PAN) by Churg and Strauss in 1951, like a systemic and pulmonary vasculitis characterized by the existence of severe asthma, blood and tissue hyper eosinophilia, associated with visceral disorders similar to those of PAN [3]. Subsequently, it was defined and classified according to the Chapel Hill consensus conference on the nomenclature and classification of systemic vasculitides of 1992 revised in 2012, as small and medium-sized vessel vasculitis, associating: eosinophil-rich granulomatous inflammation of respiratory tract, necrotizing systemic vasculitis, asthma, and eosinophilia [4,5].

It is also an autoimmune vasculitis mediated by antineutrophil cytoplasmic antibodies (ANCA) (ANCA-associated vasculitis) [6]. These autoantibodies are noted in about $2 / 3$ of cases, usually of perinuclear pattern (pANCA), and with anti-myeloperoxidase specificity (anti-MPO) [1,2,6]. This systemic angiitis is very rare with an overall prevalence estimated at $10.7-14$ per 1 million and an incidence of approximately $0.11-2.66$ per 1 million people per year [7]. Its diagnosis remains, however, a real diagnostic challenge for clinicians, particularly in atypical and incomplete forms, forms with unusual localizations, or forms without ANCA $[1,2]$. Indeed, recent genome-wide association studies suggests the presence of two genetically distinct subgroups/phenotypes of EGPA: ANCA-positive and -negative subgroups [1,2]. Endocrine damage particularly that of the thyroid gland, are exceptional during systemic vasculitis [8-13]. They raise the problem of their mechanism (association of autoimmune diseases? Or specific thyroid localization of vasculitis?), As well as that of their treatment and prognostic implications [8-13].

During EGPA, thyroid involvement is exceptional and can theoretically result from two mechanisms:

1. A specific mechanism of the disease either by necrotizing vasculitis of the thyroid arterioles or by eosinophilic infiltration of the thyroid parenchyma by analogy to the other systemic manifestations of the disease. We can compare this mechanism to that of the case of Maltzahn M. et al: pseudo-tumor ovarian involvement caused by necrotizing and eosinophilic ovarian vasculitis proven histologically in an 81-year-old woman followed for EGPA [14].

i. An association of autoimmune diseases: EGPA and autoimmune thyroiditis (Hashimoto thyroiditis or Graves's disease) due to the common dysimmune denominator of the 
two conditions. This mechanism is illustrated by the observation of Sagara $\mathrm{Y}$ et al, reporting autoimmune thyroiditis and type 1 diabetes in a 60-year-old woman occurring 12 years after the initial diagnosis of granulomatosis with polyangiitis. This patient expressed simultaneously positive anti-GAD autoantibodies (specific for autoimmune diabetes), positive anti-TPO (specific for Hashimoto's thyroiditis), and positive pANCA (specific for EGPA) [15]. This hypothesis is also reinforced by the association of EGPA with other autoimmune diseases such as rheumatoid arthritis and autoimmune hepatitis $[16,17]$.

\section{References}

1. Furuta S, Iwamoto T, Nakajima H (2019) Update on eosinophilic granulomatosis with polyangiitis. Allergol Int 68(4): 430-436.

2. Wu EY, Hernandez ML, Jennette JC, Falk RJ (2018) Eosinophilic Granulomatosis with Polyangiitis: Clinical Pathology Conference and Review. J Allergy Clin Immunol Pract 6(5): 1496-1504.

3. Churg J, Strauss L (1951) Allergic granulomatosis, allergic angiitis, and periarteritis nodosa. Am J Pathol 27(2): 277-301.

4. Holl-Ulrich K (2014) Vasculitis: New nomenclature of the Chapel Hill consensus conference 2012. Pathologe 34(6): 569-579.

5. Mouthon L, Dunogue B, Guillevin L (2014) Diagnosis and classification of eosinophilic granulomatosis with polyangiitis (formerly named Churg-Strauss syndrome) J Autoimmun 48-49: 99-103.

6. Yates M, Watts R (2017) ANCA-associated vasculitis. Clin Med (Lond) 17(1): 60-64.

7. Greco A, Rizzo MI, De Virgilio A, Gallo A, Fusconi M, et al. (2015) ChurgStrauss syndrome. Autoimmun Rev 14(4): 341-348.
8. Bouomrani S (2018) Thyroid Disorders in Systemic Angiitis: Myth or Reality?. EC Endocrinology and Metabolic Research 3(3): 133-135.

9. Bouomrani S, Ben Hamad M, Regaieg N, Belgacem N, Trabelsi S (2018) Endocrine involvement in granulomatosis with polyangiitis. Res Rev Insights 2(2): 1-3.

10. Bouomrani S, Trabelsi S, Halwani C, Ben Hamad M, Regaïeg N, et al. (2018) Thyroid Involvement in Takayasu's Disease. Rep Thyroid Res 2(1): 105.

11. Bouomrani S, Trabelsi S (2019) Hypothyroidism in Microscopic Polyangiitis. JMCRR 2-3: 164-170.

12. Bouomrani S, Tabelsi S, Guermazi (2019) Thyroid gland involvement in Henoch-Schönlein purpura. Int J Thyro Disord Ther 1(1): 1-5.

13. Bouomrani S, Trabelsi S, Guermazi M, Regaieg N. (2019) Thyroid Involvement In Behçet's Disease. Int J Thyro Disord Ther 1(1): 1-7.

14. von Maltzahn M, Kinloch M, Truchan M, Taylor-Gjevre RM (2014) Vasculitic adnexal involvement in eosinophilic granulomatosis with polyangiitis (Churg-Strauss angiitis). J Clin Rheumatol 20(6): 341-342.

15. Sagara Y, Tajikubo M, Hayashi H, Akazawa S (2008) A case of type 1 diabetes mellitus coexisting with autoimmune thyroiditis disease occurring 12 years after Churg-Stauss syndrome onset. J Japan Diab Soc 51(11): 1007-1012.

16. Szilasi M, Mátyus J, File I, Szücs G, Rákóczi E, Pfliegler G, et al. (2012) Association of ANCA-associated vasculitis-rheumatoid arthritis overlap syndrome in four patients: rituximab may be the right choice? Autoimmunity 45(4): 304-309.

17. Lohani S, Nazir S, Tachamo N, Pagolu P (2017) Autoimmune hepatitis and eosinophilic granulomatosis with polyangiitis: a rare association. BMJ Case Rep 2017: bcr2016218385.

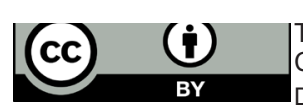

This work is licensed under Creative Commons Attribution 4.0 Licens

DOI: 10.19080/JETR.2020.05.555667 